\title{
A randomized trial to determine the diagnostic accuracy of conventional vs. jumbo forceps biopsy of gastric epithelial neoplasias before endoscopic submucosal dissection; open-label study
}

\author{
Hyo Keun Jeon · Ho Yoel Ryu $\cdot$ Mee Yon Cho $\cdot$ Hyun-Soo Kim $\cdot$ \\ Jae Woo Kim · Hong Jun Park · Moon Young Kim • Soon Koo Baik • \\ Sang Ok Kwon $\cdot$ Su Yeon Park $\cdot$ Sung Ho Won
}

Received: 9 August 2013/ Accepted: 24 November 2013/Published online: 13 December 2013

(c) The International Gastric Cancer Association and The Japanese Gastric Cancer Association 2013

\begin{abstract}
Background and aims Larger biopsy specimens or increasing the number of biopsies may improve the diagnostic accuracy of gastric epithelial neoplasia (GEN). The aims of this study was to compare the diagnostic accuracies between conventional and jumbo forceps biopsy of GEN before endoscopic submucosal dissection (ESD) and to confirm that increasing the number of biopsies is useful for the diagnosis of GEN.

Results The concordance rate between EFB and ESD specimens was not significantly different between the two groups $[83.1 \%(54 / 65)$ in JG vs. $79.1 \%(53 / 67)$ in CG]. On multivariate analyses, two or four EFBs significantly increased the cumulating concordance rate [coefficients; twice: $5.1(P=0.01)$, four times: $5.9(P=0.02)]$. But, the concordance rate was decreased in high grade dysplasia (coefficient $-40.32, P=0.006$ ).

Patients and methods One hundred and sixty GENs from 148 patients were randomized into two groups and finally
\end{abstract}

H. K. Jeon and H. Y. Ryu equally contributed to this work.

H. K. Jeon · H. Y. Ryu · H.-S. Kim $(\bowtie)$.

J. W. Kim - H. J. Park - M. Y. Kim - S. K. Baik · S. O. Kwon Division of Gastroenterology and Hepatology, Department of Internal Medicine, Yonsei University Wonju College of

Medicine, 162 Ilsan-dong, Wonju, Gangwon-do 220-701,

Republic of Korea

e-mail: hyskim@yonsei.ac.kr

\section{Y. Cho}

Department of Pathology, Yonsei University Wonju College of Medicine, Wonju, Republic of Korea

S. Y. Park · S. H. Won

Department of Applied Statistics, Chung-Ang University, Seoul, Republic of Korea
67 GENs in 61 patients and 65 GENs in 63 patients were allocated to the conventional group (CG) or jumbo group (JG), respectively. Four endoscopic forceps biopsy (EFB) specimens were obtained from each lesion with conventional $(6.8 \mathrm{~mm})$ forceps or jumbo $(8 \mathrm{~mm})$ forceps. The histological concordance rate between 4 EFB specimens and ESD specimens was investigated in the two groups. Conclusions Before ESD, the diagnostic accuracy of GENs was significantly increased not by the use of jumbo forceps biopsy but by increasing the number of biopsies.

Keywords Gastric epithelial neoplasia - Diagnosis · Endoscopic forceps biopsy · Endoscopic submucosal dissection

\section{Introduction}

Endoscopic forceps biopsy (EFB) is essential for the histological diagnosis of gastric epithelial neoplasia (GEN). However, discrepancies between an EFB and subsequent resection specimens have frequently been noted in a clinical setting [1-5]. An EFB does not represent the entire lesion because only a small portion of the lesion is sampled using this technique. Therefore, an EFB may underestimate gastric epithelial neoplastic lesions and lead to undertreatment.

According to the Vienna classification, the practical recommendation for low-grade dysplasia (LGD) is initially a surveillance or a local treatment such as endoscopic resection, whereas the recommendation for high-grade dysplasia (HGD) is local resection [6, 7]. The natural course of HGD involves frequent malignant transformation, whereas low-grade dysplasia (LGD) progression to malignancy is less likely [8-10]. Therefore, a precise 
histological diagnosis is crucial for planning appropriate therapeutic action for dysplastic lesions.

Recently, endoscopic submucosal dissection (ESD) has become a commonly used technique for gastric dysplasia, and therapeutic outcomes of en block resection are very promising regardless of lesion size and location. However, the application of ESD for all gastric dysplastic lesions may increase the chance of potentially serious complications of bleeding or perforation as well as time and cost of care without proven long-term benefits [10-12]. In Korea, national health insurance strictly regulates the ESD indications and does not reimburse the fee per performance on patients with low grade dysplasia less than $3 \mathrm{~cm}$ in size, because appropriately diagnosed low grade dysplasia is not necessarily removed. Therefore, an estimation of the diagnostic yield of EFB and judging the grade of GEN using EFB before ESD in GEN is essential to avoid unnecessary therapeutic procedures and complications. An accurate pathologic diagnosis through EFB can be obtained by using an increased number of samples or sampling a greater surface area. This proposition has been demonstrated in Barrett's esophagus and inflammatory bowel disease, where jumbo forceps are recommended for collecting surveillance biopsy specimens [13-15]. In the study, we prospectively investigated the diagnostic accuracy of EFB using $8.0 \mathrm{~mm}$ jumbo forceps compared with $6.8 \mathrm{~mm}$ conventional forceps for GEN before ESD and the usefulness of increasing the number of biopsies for the diagnosis of GEN.

\section{Methods}

\section{Patients}

From June 2009 to November 2010 we prospectively recruited 148 patients with 160 GENs. All of the lesions were pathologically proven or suspected as GENs on EFB. Patients were included in the study according to the following criteria: (1) older than the age of $18,(2)$ more than $1 \mathrm{~cm}$ sized GENs estimated by chromoendoscopy with indigo carmine or narrow band imaging (NBI) and (3) provision of informed consent. Patients were excluded from the study if they met the following criteria: (1) gastric epithelial neoplastic lesions including ulceration or scarring and (2) unable or unwilling to provide informed consent. This study was approved by Institutional Review Board (IRB) for Human Research Yonsei University Wonju College of Medicine. IRB approval number was CR309003 and the approval date was June 24, 2009.

One hundred and sixty GENs from 148 patients were randomized into conventional group (CG, $n=83)$ vs. jumbo group (JG, $n=77$ ), using an envelope method by the research assistant in third room. Patients, endoscopists who performed ESD and the pathologist were blinded, but endoscopists who perform EFBs were opened. To minimize the potential bias from the forceps, the endoscopist did not recognize the forceps type until the research assistant provided the forceps during the endoscopic procedure. Of the 148 patients, ESD was not performed in 15 because 7 patients did not report for their scheduled ESD and 8 referred patients were not pathologically confirmed as GEN on EFB.

A total of 145 lesions were resected by ESD. However, 13 lesions were excluded; 2 lesions were finally diagnosed as intestinal metaplasia after ESD, even though a previous EFB resulted in a diagnosis of GEN at our hospital. These cases may include small neoplastic lesions that might have been completely removed by the previous forceps biopsy [16] because no evidence of GEN was found on the followup endoscopy 1 month after ESD. Another 8 lesions which was initially diagnosed as an equivocal lesion such as indefinite dysplasia or regenerative atypia by EFB were finally diagnosed as metaplasias after ESD. The other three lesions were also excluded because of an inadequate number of biopsy specimens obtained by EFB. In total, 132 GENs from 122 patients were finally analyzed; the CG included 68 GENs whereas the JG included 65 GENs.

\section{Biopsy forceps}

The CG in this study consisted of patients for whom EFB was performed using conventional elongated cups and a fenestrated needle with an open diameter of $6.8 \mathrm{~mm}$ (FB24K-1; Olympus Optical Co., Ltd, Tokyo, Japan). The JG included patients who were endoscopically biopsied with non-spiked biopsy forceps with an open diameter of $8 \mathrm{~mm}$ (Cook Medical, Bloomington, USA). Four specimens were obtained from each lesion. The first biopsy was performed on the most suspicious site for GEN, because bleeding after the first biopsy might cover the lesion and it would be difficult to obtain the adequate specimens on the next biopsies. EFB specimen was labeled with a unique study code number. A single expert gastrointestinal pathologist was blinded to the clinical data examined all the specimens. The ESD specimens were sent to the pathologist after the biopsy results were received.

\section{ESD procedure}

We performed ESD within 4 weeks after obtaining the four EFB specimens. All procedures were performed using a conventional single-channel endoscope (GIF-Q260; Olympus Optical Co., Ltd, Tokyo, Japan) and an electrocauterizer (VIO 300D, ERBE, Tübingen, Germany) with the patients under conscious sedation. All of the gastric 
neoplastic lesions were resected by the standard ESD technique using a hook knife (KD-611L; Olympus Optical Co., Ltd., Tokyo, Japan) or flex knife (KD-603L; Olympus) alone or in combination with the IT-2 knife (KD-611L; Olympus), depending on the endoscopic features and histological characteristics of the lesions [11, 17].

\section{Histological evaluation}

All biopsy specimens were fixed in $10 \%$ formaldehyde solution and embedded in paraffin. The paraffin blocks were cut into $4-\mu \mathrm{m}$-thick sections for hematoxylin-eosin (H\&E) staining. Endoscopically resected specimens were washed in normal saline, oriented on a corkboard with small pins, fixed in $10 \%$ formaldehyde solution, and embedded in paraffin. The specimens were sectioned serially at 2-mm intervals and then submitted for histologic diagnosis. One expert pathologist reviewed the specimens taken by forceps biopsy and ESD. The pathologist was blinded to the biopsy results when she read the ESD specimen.

The histological diagnosis was determined according to the World Health Organization classification [18] and the guidelines of the Gastrointestinal Pathology Study Group of the Korean Society of Pathologists for differential diagnosis: (1) a diagnosis of carcinoma is based on invasion; (2) the most important characteristic of low grade dysplasia is the architectural pattern such as regular distribution of crypts without severe branching, budding, or marked glandular crowding; (3) if nuclear pseudostratification occupies more than the basal half of the cryptal cells in three or more adjacent crypts, the lesion is considered high grade dysplasia; (4) if severe cytologic atypia is present, careful inspection for invasive foci is necessary, because the risk for invasion is very high; (5) other structural or nuclear atypia should be evaluated to make a final decision such as cribriform pattern, papillae, ridges, vesicular nuclei, high nuclear/cytoplasmic ratio, loss of nuclear polarity, thick and irregular nuclear membrane, and nucleoli [19].

Each of the four EFB specimens was compared with the corresponding ESD specimens, and we measured EFB specimens to determine the (1) length in millimeters and (2) depth in millimeters. EFB specimens sampled perpendicularly were measured along the long and short axes.

\section{Statistical analysis}

The primary outcome was the fraction of concordant biopsy specimens obtained by the jumbo forceps with ESD specimens, compared with that by the conventional forceps.
To calculate the statistical power, we made a few assumptions: first, the diagnostic accuracy of conventional forceps biopsy might be $55.5 \%$ based on previous study reference [3]. Second, specimens might be in round shape by using conventional or jumbo forceps and the surface area of each specimen could be calculated by $\pi$ (radius) ${ }^{2}$. Therefore, the jumbo forceps might obtain a specimen $38.4 \%$ wider in surface area than those obtained by conventional forceps theoretically. Third, considering the area difference of $38.4 \%$, the diagnostic accuracy of jumbo forceps biopsy would be $21 \%$ higher than that of conventional forceps biopsy relatively.

According to above assumptions, power calculations showed that a sample size of 65 GENs per group would provide $66 \%$ power to detect a $21 \%$ difference in proportions of the diagnostic accuracy relatively, using a twogroup Fisher's exact test with a 2 -sided $P$ value of 0.05 . All analyses relative to the sample size calculation were performed with $G^{*}$ Power version 3.1 program [20, 21].

We also investigated the cumulative concordance rate between the first through the fourth EFB specimens and the ESD specimen in both groups.

Univariate analysis was performed using Student's $t$ test for the continuous variables. The $\chi^{2}$ test employing Yates' correction for continuity where appropriate was used for the categorical data. If each cell in $2 \times 2$ contingency table data had expected count less than 5, Fisher's exact test was used in the analysis.

Ten parameters in multivariate analysis with the use of generalized linear mixed model included clinical factors to be potentially associated with the histological concordance between EFBs and the ESD specimen. The fitness of multivariate model was evaluated by the Likelihood-ratio test and a random effect was considered in the model. Coefficients of variables for multivariate model were estimated by using Wald test. We considered the correlation of estimates and there were no highly correlated variables included in multivariate model. The statistical significance was defined as $P<0.05$. Statistical analysis was performed using SPSS-PC 13.0 (SPSS Inc., Chicago, IL, USA) and R statistical program 2.15.1 (http://cran.r-project.org/).

\section{Results}

Baseline characteristics

The mean age of the enrolled patients was 66 years (range 40-86 years). There were no significant differences in age, gender, endoscopic features, distribution or the degree of dysplasia between the two groups. The characteristics of the patients in the two groups are shown in Table 1. 
Table 1 Baseline characteristics and endoscopic features in the conventional forceps and jumbo forceps groups

\begin{tabular}{|c|c|c|c|c|}
\hline & Conventional forceps $(n=67)$ & Jumbo forceps $(n=65)$ & Total $(n=132)$ & $P$ value \\
\hline Age (mean $\pm \mathrm{SD}$, years) & $66.1 \pm 9.4$ & $64.7 \pm 10.1$ & $65.4 \pm 9.7$ & 0.44 \\
\hline Males, no. (\%) & $45(73.8)$ & $47(77.0)$ & $92(75.4)$ & 0.67 \\
\hline \multicolumn{5}{|l|}{ Endoscopic feature, no. (\%) } \\
\hline Median size of specimen (mm) & $16.7 \pm 9.9$ & $16.3 \pm 12.5$ & $16.5 \pm 11.2$ & 0.81 \\
\hline Macroscopic type & & & & 0.25 \\
\hline Elevated & $26(38.8)$ & $34(52.3)$ & $60(45.5)$ & \\
\hline Flat & $28(41.8)$ & $23(35.4)$ & $51(38.6)$ & \\
\hline Depressed & $13(19.4)$ & $8(12.3)$ & $21(15.9)$ & \\
\hline Location of lesion & & & & 0.12 \\
\hline Upper & $4(6.0)$ & $2(3.1)$ & $6(4.5)$ & \\
\hline Middle & $15(22.4)$ & $25(38.5)$ & $40(30.3)$ & \\
\hline Lower & 48 (71.6) & $38(58.5)$ & $86(65.2)$ & \\
\hline Surface color & & & & 0.30 \\
\hline White to yellow & $44(65.7)$ & $48(73.8)$ & $92(69.7)$ & \\
\hline Erythematous red & $23(34.3)$ & $17(26.2)$ & $40(30.3)$ & \\
\hline Surface nodularity & & & & 0.14 \\
\hline Nodular & $14(20.9)$ & $21(32.3)$ & $35(26.5)$ & \\
\hline Smooth & $53(79.1)$ & $44(67.7)$ & $97(73.5)$ & \\
\hline Endoscopist experience $^{\mathrm{a}}$ & & & & 0.30 \\
\hline Non-expert & $28(41.8)$ & $33(50.8)$ & $61(46.2)$ & \\
\hline Expert & $39(58.2)$ & $32(49.2)$ & $71(53.8)$ & \\
\hline
\end{tabular}

a Beginner was defined as having 6 months-2 years of endoscopy experience and expert was defined has having more than 2 years of endoscopy experience

Specimen size assessment

All specimens were measured in millimeters along both the long and short axes. The mean $( \pm \mathrm{SD})$ diameter of the EFB taken with the conventional forceps was $2.48( \pm 0.53) \mathrm{mm}$, whereas that with the jumbo forceps was $2.89( \pm 0.60) \mathrm{mm}$. The difference in diameter was statistically significant $(P<0.01)$.

Pathologic results and histological concordance rate between EFB and ESD specimens

The pathologic results from EFB specimens obtained using conventional or jumbo forceps are shown in Table 2. The overall histological concordance rate between the EFB including jumbo forceps and conventional forceps and ESD specimens was $81.1 \%(107 / 132)$ among the enrolled patients (Table 3 ). The cumulating concordance rate including 4 biopsy specimens was not significantly higher in the jumbo forceps biopsy group than the CG [83.1\% (54/65) vs. $79.1 \%(53 / 67), P=0.56)$. Also, the first biopsy was not significantly different between JG [70.8\% (46/65)] and CG [65.7\% (44/67), $P=0.53]$. Repeated endoscopic biopsies to 4 times gradually increased the
Table 2 Final pathological results for conventional and jumbo endoscopic forceps biopsy specimens

\begin{tabular}{llll}
\hline & $\begin{array}{l}\text { Conventional } \\
\text { forceps, } n(\%) \\
(n=67)\end{array}$ & $\begin{array}{l}\text { Jumbo forceps, } \\
n(\%) \\
(n=65)\end{array}$ & $\begin{array}{l}\text { Total, } \\
n(\%) \\
(n=132)\end{array}$ \\
\hline $\begin{array}{c}\text { Low grade } \\
\text { dysplasia }\end{array}$ & $24(35.8)$ & $30(46.2)$ & $54(40.9)$ \\
$\begin{array}{c}\text { High grade } \\
\text { dysplasia }\end{array}$ & $11(16.4)$ & $8(12.3)$ & $19(14.4)$ \\
$\begin{array}{c}\text { Adenocarcinoma } \\
\text { Indefinite } \\
\text { dysplasia }\end{array}$ & $30(44.8)$ & $26(40.0)$ & $56(42.4)$ \\
$\begin{array}{c}\text { Intestinal } \\
\text { metaplasia }\end{array}$ & $1(1.5)$ & $1(1.5)$ & $2(1.5)$ \\
\hline
\end{tabular}

cumulating concordance rate between EFB and ESD specimen (Fig. 1).

Factors related to the histological concordance between EFB and ESD specimens

On multivariate analysis, two or four EFBs significantly increased the cumulating concordance rate (coefficients; twice: $5.1(P=0.01)$, four times: $5.9(P=0.02)$, Table 4$)$. 
Table 3 Comparisons between the histological results of EFB and ESD specimens $[n(\%)]$

\begin{tabular}{llllr}
\hline Endoscopic biopsy & \multicolumn{4}{l}{ Endoscopic submucosal dissection } \\
\cline { 2 - 5 } & LGD & HGD & Adenocarcinoma & Total \\
\hline LGD & & & & \\
$\quad$ Conventional forceps & $19(79.2)$ & $4(16.7)$ & $1(4.1)$ & 24 \\
$\quad$ Jumbo forceps & $23(76.7)$ & $5(16.7)$ & $2(6.6)$ & 30 \\
HGD & & & & \\
Conventional forceps & $1(9)$ & $5(45.5)$ & $5(45.5)$ & 11 \\
Jumbo forceps & $2(25)$ & $5(62.5)$ & $1(12.5)$ & 8 \\
Adenocarcinoma & $0(0)$ & $1(1.8)$ & $55(98.2)$ & 56 \\
Indefinite dysplasia & $0(0)$ & $1(50.0)$ & $1(50.0)$ & 2 \\
Intestinal metaplasia & $1(100)$ & 0 & 0 & 1 \\
Total & 46 & 21 & 65 & 132 \\
\hline
\end{tabular}

$L G D$ low grade dysplasia, $H G D$ high grade dysplasia

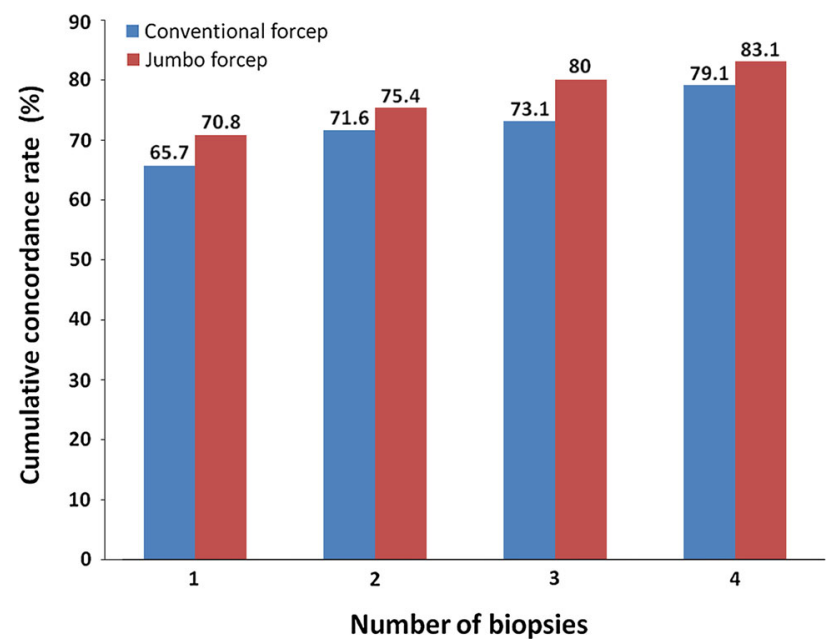

Fig. 1 Cumulative concordance rates between EFB and ESD specimens were compared for the conventional forceps and jumbo forceps group

Three EFBs also tended to increase the cumulating concordance rate (coefficient $2.44, P=0.11$ ) with marginal significance. Interestingly, the concordance rate between the EFB and ESD specimens was dependent on the degree of dysplasia on EFB. For LGD, each concordance rate between the first through the fourth EFB specimens and the ESD specimen was relatively high; 76.2, 81, 81 and $95.2 \%$ in the CG and 84, 92, 96 and $96 \%$ in the JG. In HGD, the concordance rates between the first through the fourth EFB specimens and the ESD specimen were low; $40 \%$ for all in the CG and $45.5 \%$ for all in the JG. In particular, compared to LGD, the histological concordance rate between EFB and ESD specimens was significantly decreased in HGD on multivariate model (coefficient $-40.32, P=0.01$ ). In cases of adenocarcinoma on initial $\mathrm{EFB}$, the concordance rates between the
Table 4 Multivariate analysis for factors associated with histological concordance rate between EFBs and ESD specimens

\begin{tabular}{|c|c|c|c|c|}
\hline & Coefficient & $\begin{array}{l}\text { Standard } \\
\text { error }\end{array}$ & $z$ value & $P$ value \\
\hline $\begin{array}{r}\text { Fixed effect } \\
\text { (Intercept) }\end{array}$ & -1.46 & 164.92 & -0.009 & 0.99 \\
\hline \multicolumn{5}{|l|}{ Types of forceps } \\
\hline \multicolumn{5}{|l|}{ Conventional forcep } \\
\hline Jumbo forcep & -0.38 & 8.17 & -0.046 & 0.96 \\
\hline \multicolumn{5}{|l|}{ Number of biopsies } \\
\hline \multicolumn{5}{|l|}{ One } \\
\hline Two & 5.10 & 1.98 & 2.581 & 0.01 \\
\hline Three & 2.44 & 1.53 & 1.59 & 0.11 \\
\hline Four & 5.94 & 2.54 & 2.34 & 0.02 \\
\hline Age & 0.96 & 5.01 & 0.19 & 0.85 \\
\hline $\mathrm{Age}^{2}$ & -0.01 & 0.04 & -0.22 & 0.83 \\
\hline \multicolumn{5}{|l|}{ Sex } \\
\hline \multicolumn{5}{|l|}{ Male } \\
\hline Female & 0.12 & 10.49 & 0.01 & 0.99 \\
\hline \multicolumn{5}{|l|}{ Endoscopist experience } \\
\hline \multicolumn{5}{|l|}{ Non-expert } \\
\hline Expert & 2.42 & 8.82 & 0.27 & 0.78 \\
\hline Size $(\mathrm{mm})$ & 0.35 & 0.37 & 0.95 & 0.34 \\
\hline \multicolumn{5}{|l|}{ Macroscopic type } \\
\hline \multicolumn{5}{|l|}{ Elevated } \\
\hline Flat & -12.22 & 10.40 & -1.17 & 0.24 \\
\hline Depressed & 6.55 & 14.02 & 0.47 & 0.64 \\
\hline \multicolumn{5}{|l|}{ Location } \\
\hline \multicolumn{5}{|l|}{ Upper } \\
\hline Middle & 14.84 & 29.09 & 0.51 & 0.61 \\
\hline Lower & 12.67 & 28.53 & 0.44 & 0.66 \\
\hline \multicolumn{5}{|l|}{ Surface color } \\
\hline \multicolumn{5}{|l|}{ Erythematous } \\
\hline White to yellow & -18.02 & 11.68 & -1.54 & 0.12 \\
\hline \multicolumn{5}{|l|}{ Pathology after ESD } \\
\hline \multicolumn{5}{|l|}{ Low grade dysplasia } \\
\hline High grade dysplasia & -40.32 & 14.60 & -2.76 & 0.01 \\
\hline Carcinoma & -20.70 & 12.72 & -1.63 & 0.10 \\
\hline \multicolumn{5}{|l|}{ Surface nodularity } \\
\hline \multicolumn{5}{|l|}{ Smooth } \\
\hline Nodular & -0.91 & 10.69 & -0.09 & 0.93 \\
\hline \multicolumn{5}{|l|}{ Random effect } \\
\hline Var (ID) & 602.18 & 24.54 & - & - \\
\hline
\end{tabular}

first through the fourth EFB specimens and the ESD specimen were $66.7,75,77.8$ and $80.6 \%$ in CG and 69, $72.4,79.3$ and $86.2 \%$ in the JG. However, other parameters such as size, endoscopic morphology, location and forceps type were not associated with histological concordance or discrepancy (Tables 4,5 ). 
Table 5 Univariate analyses for factors associated with histological discrepancy between EFBs and ESD specimens $(n=132)$

\begin{tabular}{|c|c|c|c|}
\hline Factors & $\begin{array}{l}\text { Discrepancy } \\
\text { (no. cases/ } \\
\text { total) }\end{array}$ & $\begin{array}{l}\text { Univariate } \\
\text { analysis } \\
\text { OR }(95 \% \mathrm{CI})\end{array}$ & $P$ value \\
\hline \multicolumn{4}{|l|}{ Size } \\
\hline$<2 \mathrm{~cm}$ & $16 / 89$ & 1.0 (reference) & \\
\hline$\geq 2 \mathrm{~cm}$ & $9 / 43$ & $1.21(0.48-3.01)$ & 0.68 \\
\hline \multicolumn{4}{|l|}{ Macroscopic type } \\
\hline Elevated & $9 / 60$ & 1.0 (reference) & \\
\hline Flat & $15 / 51$ & $2.36(0.93-5.98)$ & 0.07 \\
\hline Depressed & $1 / 21$ & $0.28(0.03-2.38)$ & 0.24 \\
\hline \multicolumn{4}{|l|}{ Location } \\
\hline Upper & $2 / 6$ & 1.0 (reference) & \\
\hline Middle & $6 / 40$ & $0.35(0.05-2.37)$ & 0.28 \\
\hline Lower & $17 / 86$ & $0.49(0.08-2.91)$ & 0.43 \\
\hline \multicolumn{4}{|l|}{ Surface color } \\
\hline Erythematous & $5 / 40$ & 1.0 (reference) & \\
\hline White to yellow & $20 / 92$ & $1.94(0.67-5.61)$ & 0.21 \\
\hline \multicolumn{4}{|l|}{ Surface nodularity } \\
\hline Smooth & $18 / 97$ & 1.0 (reference) & \\
\hline Nodular & $7 / 35$ & $1.09(0.41-2.91)$ & 0.85 \\
\hline \multicolumn{4}{|c|}{ Pathology after ESD } \\
\hline $\begin{array}{r}\text { Low grade } \\
\text { dysplasia }\end{array}$ & $2 / 46$ & 1.0 (reference) & \\
\hline $\begin{array}{r}\text { High grade } \\
\text { dysplasia }\end{array}$ & $12 / 21$ & $\begin{array}{l}29.33 \\
\quad(5.57-154.24)\end{array}$ & $<0.05$ \\
\hline Carcinoma & $11 / 65$ & $4.48(0.94-21.29)$ & 0.05 \\
\hline \multicolumn{4}{|c|}{ Endoscopist experience ${ }^{a}$} \\
\hline Non-expert ${ }^{\mathrm{a}}$ & $14 / 61$ & 1.0 (reference) & \\
\hline Expert & $11 / 71$ & $0.61(0.25-1.48)$ & 0.27 \\
\hline \multicolumn{4}{|l|}{ Types of forceps } \\
\hline $\begin{array}{l}\text { Conventional } \\
\text { forceps }\end{array}$ & $13 / 66$ & 1.0 (reference) & \\
\hline Jumbo forceps & $10 / 65$ & $0.74(0.29-1.83)$ & 0.51 \\
\hline
\end{tabular}

$C I$ confidence interval, $E F B$ endoscopic forceps biopsy, ESD endoscopic submucosal dissection, $O R$ odds ratio

a Non-expert was defined has having 6 months-2 years of endoscopy experience and expert was defined as having more than 2 years of endoscopy experience

\section{Discussion}

Theoretically, the larger the portion of dysplasia can be sampled due to the endoscopy biopsy forceps with the large cup, thus increasing the diagnostic accuracy. Jumbo forceps improve the diagnostic accuracy of dysplasia in Barrett's esophagus and inflammatory bowel disease [13-15]. However, the diagnostic accuracy of the jumbo forceps was not significantly higher than that of conventional forceps in the study. There could be several explanations for the negative results. First, the discrepancy rate between EFB specimens and the resected specimen in the study was lower than that of previous studies [1-5]. We had expected that the discrepancy rate of conventional forceps would be $44.5 \%$. However, it was only $20.9 \%$ in all 4 EFB specimens and the ESD specimen. We failed to make a theoretical $21 \%$ gain in diagnostic accuracy from jumbo forceps, because the diagnostic accuracy of the conventional forceps was higher than expected. This result could be regarded as a ceiling effect. Second, most patients included in the study were transferred to our tertiary endoscopic center with documented lesion characteristics before EFB. This information likely enabled the endoscopists to perform the target biopsy which is critical for getting the adequate specimens for the diagnosis of GEN, regardless of the forceps type. This reasoning was supported by the result that the gain of diagnostic yield from additional 3 biopsies in the jumbo group $(12.3 \%)$ was not significantly different than that in the conventional group (13.4\%). Third, advantages of jumbo forceps with enlarged cups were not fully recognized because of the relatively small size of the lesion in the study. Additional biopsies could be performed on the small sized or the most suspicious area of the lesions, resulting in veiling the beneficial effect of jumbo forceps. Fourth, the average thickness of the mucosa is around $1 \mathrm{~mm}$ in normal mucosae or flat lesions. Therefore, both jumbo and conventional biopsy forceps can cover the whole thickness of the mucosal layer. EFB specimen obtaining from jumbo forceps can cover a wider area of mucosa, but it did not reach deeper layer than that from conventional forceps. In case of elevated mucosal lesions such as the EGC type I or IIa, the invasive cancer cells may reside in the deepest part of the mucosa. Since most EFBs get specimens around $1 \mathrm{~mm}$ in depth, the chance of missing the invasive cancer cells in the lesion occurs and this may contribute to the diagnostic discrepancy between EFB and final ESD specimens. Therefore, this result could support that jumbo forcep biopsy specimens had no advantage of increasing the concordance rate. Fifth, despite most parts of the lesion may be of low grade nature, the final diagnosis is made by the highest grade of the lesion even if it is focal or minute. Therefore, the discrepancy between EFB and ESD diagnosis cannot completely be explained by the amount of tissues obtained by forceps, because of non-random and scattered distribution of the highest grade lesions. In addition, there are chances of missing the highest grade area regardless of the type of biopsy forceps if EFB is randomly taken.

In the result, the cumulative diagnostic accuracy of four endoscopic biopsies was significantly improved by about 12-14\% compared to that of only one biopsy before ESD in both groups. These results were different compared to those from previous studies showing no additional benefits by increasing the number of forceps biopsies taken [3, 4]. 
Compared to the previous retrospective studies, this study had strengths because the benefits of additional biopsies were proven by prospective design. Furthermore, a recent report [22] supported these results, even though it was performed in advanced gastric cancer. These findings suggest that obtaining four EFB samples benefit the selection of an appropriate treatment strategy in patients with GENs.

The Vienna classification recommends two treatment options for LGD, local resection or follow-up [6, 7]. Generally, many clinicians are comfortable with a routine endoscopic follow-up if the lesion is truly LGD. However, the EFB may not represent the entire lesion, and this can result in underestimation of the possibility of co-existing HGD or cancer [23]. Also, interobserver variation in the diagnosis of LGD is possible [1, 6, 7, 23]. In addition, endoscopic mucosal resection including ESD should be widely considered for large lesions or lesions containing a depressed region because these features are closely related to hidden malignancy or histologic progression noted after resection [23]. Our study showed that all four EFBs increased the concordance rate with ESD specimens from $76.2 \%$ for the first EFB to $95.2 \%$ in LGD. These results suggest that at least four EFBs should be performed in order to provide more accurate histological results before ESD.

According to the WHO classification, HGD is diagnosed when architectural distortion is increased with glandular crowding and prominent cellular atypia without stromal invasion $[6,7,18]$. Carcinoma is diagnosed when the lamina propria or the muscularis mucosa is involved [6, 7, 18]. In our experience, a high proportion of HGD based on EFB was upgraded to carcinoma after ESD (Table 3). In particular, conventional forceps tended to underestimate the severity of dysplasia, compared with jumbo forceps (5/ $11(45.5 \%)$ vs. $1 / 8(12.5 \%)$ ) in the study results (Table 3 ), although it was not significant statistically. Our findings are consistent with those of previous studies [2, 3, 5], and can be explained by the fact that EFB specimens taken from an HGD lesion are too focal to represent the whole area of the lesion for adequate diagnosis. Therefore, our results support the use of complete endoscopic or surgical resection of the tumor according to current guidelines for cases of HGD.

An accurate pathologic diagnosis through EFB can be obtained by using the larger surface area of samples or an increased number of samples. Although increasing the number of forceps biopsy is a simple and easy way to get more tissues, this strategy may cause the modification of neoplastic lesion through the tissue injuries in patients who are supposed to be treated by ESD. The presence of a scar was reported to affect the difficulty of ESD and the success rate of en block resection [24, 25]. EFB of a lesion can often lead to submucosal fibrosis; however, submucosal fibrosis after EFB is mild in most cases. Furthermore, the recent development of new knives may improve the success rate of en block resection, even in fibrotic lesions. In this study, lesions in cases with submucosal fibrosis were dissected under direct visualization using a hook knife or flexible knife instead of an IT-2 knife containing an insulated tip. Finally, en block resection was performed in all cases. Additionally, severe fibrosis was not observed in any cases, likely due to the fact that the ESD procedures were performed within 4 weeks after EFB.

Although, it was not statistically significant, the flat or the white to yellowish lesion was a predictive factor for histological discrepancy in this study (Table 5). Depressed lesions have been shown to be associated with a higher risk of progression to cancer $[6,23]$, and the presence of a depressed lesion or nodular lesion with erythema may result in a higher concordance rate between EFB and resected specimens because intensive target biopsies are performed for these types of lesion. However, most flat lesions do not exhibit characteristic features and this leads to random biopsies rather than a target biopsy, and random biopsies of a flat lesion may increase the histological discrepancy between the biopsy and resected specimens. Therefore, we recommend that clinicians obtain four or more EFB specimens from flat lesions in order to obtain accurate histological results before ESD, especially in lesions without depressed lesions or redness.

Several variables such as size and location of gastric neoplastic lesions and the experience of the endoscopist were not significant predictive factors for histological discrepancy. Lesion size was a significant predictor of histological discrepancy in a previous study [3], but this is inconsistent with our results. This inconsistency may be explained by the fact that there were only a few lesions greater than $2 \mathrm{~cm}$ in diameter in our study. However, lesion size was not associated with histological discrepancy in other studies $[4,5,10]$, so further study is needed to address this issue.

This study had several limitations. First, a validated outcome measure for the quality of EFB specimens in GEN does not exist. We only evaluated long and short axes without measure of penetration into the muscularis mucosa except some cases. Future study will be needed to evaluate that tissue adequacy of EFB may be related to depth of invasion. Second, although we consider that coexistence foci of HGD or cancer in the entire specimen might be related to histologic discrepancies, we did not assess the exact fraction of specific sampling errors. Therefore, further study will be needed whether the precise target biopsy using new imaging technologies such as narrow band or high definition imaging or chromoendoscopy decreases these specific sampling errors. Third, we performed 
maximum 4 endoscopic biopsies. It will be needed to verify the efficacy of more than 5 endoscopic biopsies. Fourth, only one pathologist was participated in this study, although she had a wealth of experience. Due to lack of concrete and clear-cut diagnostic criteria, moreover, the different classification and subjective diagnostic approach for dysplastic lesions [19] among pathologists may be a hurdle for the concordance as well. Two or more pathologists should confirm diagnoses of the specimens in the future study with a large sample size because a pathological diagnosis is the most important.

In summary, the size of the EFB specimens was significantly larger in the JG than in the CG. Obtaining four EFB specimens rather than only one significantly improved the diagnostic accuracy before ESD. However, the concordance rates between the EFB and the ESD specimens were not different between the CG and JG. On multivariate analyses, two or four EFBs significantly increased the cumulating concordance rate. But, the concordance rate was decreased in HGD. In conclusion, obtaining two or more EFB specimens before ESD significantly improves diagnostic reliability in comparison to just one specimen. Despite the bigger specimen size, however, jumbo forceps have not been proven to be superior to conventional forceps in terms of better diagnostic accuracy for GENs.

Acknowledgment This research was supported by Basic Science Research Program through the National Research Foundation of Korea (NRF) funded by the Ministry of Education, Science and Technology (2012-0004734).

Conflict of interest None.

\section{References}

1. Muehldorfer SM, Stolte M, Martus P, et al. Diagnostic accuracy of forceps biopsy versus polypectomy for gastric polyps: a prospective multicentre study. Gut. 2002;50:465-70.

2. Szaloki T, Toth V, Nemeth I, et al. Endoscopic mucosal resection: not only therapeutic, but a diagnostic procedure for sessile gastric polyps. J Gastroenterol Hepatol. 2008;23:551-5.

3. Lee CK, Chung IK, Lee SH, et al. Is endoscopic forceps biopsy enough for a definitive diagnosis of gastric epithelial neoplasia? J Gastroenterol Hepatol. 2010;25:1507-13.

4. Yoon WJ, Lee DH, Jung YJ, et al. Histologic characteristics of gastric polyps in Korea: emphasis on discrepancy between endoscopic forceps biopsy and endoscopic mucosal resection specimen. World J Gastroenterol. 2006;12:4029-32.

5. Sung HY, Cheung DY, Cho SH, et al. Polyps in the gastrointestinal tract: discrepancy between endoscopic forceps biopsies and resected specimens. Eur J Gastroenterol Hepatol. 2009;21: 190-5.

6. Schlemper RJ, Riddell RH, Kato Y, et al. The Vienna classification of gastrointestinal epithelial neoplasia. Gut. 2000;47:251-5.
7. Stolte M. The new Vienna classification of epithelial neoplasia of the gastrointestinal tract: advantages and disadvantages. Virchows Arch. 2003;442:99-106.

8. Rugge M, Cassaro M, Di Mario F, et al. The long term outcome of gastric non-invasive neoplasia. Gut. 2003;52:1111-6.

9. Park SY, Jeon SW, Jung MK, et al. Long-term follow-up study of gastric intraepithelial neoplasias: progression from low-grade dysplasia to invasive carcinoma. Eur J Gastroenterol Hepatol. 2008;20:966-70.

10. Jung MK, Jeon SW, Park SY, et al. Endoscopic characteristics of gastric adenomas suggesting carcinomatous transformation. Surg Endosc. 2008;22:2705-11.

11. Kato M, Nishida T, Tsutsui $\mathrm{S}$, et al. Endoscopic submucosal dissection as a treatment for gastric noninvasive neoplasia: a multicenter study by Osaka University ESD Study Group. J Gastroenterol. 2011;46:325-31.

12. Oka S, Tanaka S, Kaneko I, et al. Advantage of endoscopic submucosal dissection compared with EMR for early gastric cancer. Gastrointest Endosc. 2006;64:877-83.

13. Elmunzer BJ, Higgins PDR, Kwon YM, et al. Jumbo forceps are superior to standard large-capacity forceps in obtaining diagnostically adequate inflammatory bowel disease surveillance biopsy specimens. Gastrointest Endosc. 2008;68:273-8.

14. Gonzalez S, Yu WM, Smith MS, et al. Randomized comparison of 3 different-sized biopsy forceps for quality of sampling in Barrett's esophagus. Gastrointest Endosc. 2010;72:935-40.

15. Komanduri S, Swanson G, Keefer L, et al. Use of a new jumbo forceps improves tissue acquisition of Barrett's esophagus surveillance biopsies. Gastrointest Endosc. 2009;70:1072-8.

16. Kim ES, Jeon SW, Park SY, et al. Where has the tumor gone? The characteristics of cases of negative pathologic diagnosis after endoscopic mucosal resection. Endoscopy. 2009;41:739-45.

17. Yamamoto H. Endoscopic submucosal dissection of early cancers and large flat adenomas. Clin Gastroenterol Hepatol. 2005;3: S74-6.

18. Aaltonen LA, Hamilton SR, World Health Organization., International Agency for Research on Cancer. Pathology and genetics of tumours of the digestive system. Lyon Oxford: IARC Press; Oxford University Press (distributor; 2000).

19. Kim JM, Cho MY, Sohn JH, et al. Diagnosis of gastric epithelial neoplasia: dilemma for Korean pathologists. World J Gastroenterol. 2011;17(21):2602-10.

20. Faul F, Erdfelder E, Buchner A, Lang A-G. Statistical power analyses using $G^{*}$ Power 3.1: tests for correlation and regression analyses. Behav Res Methods. 2009;41:1149-60.

21. Faul F, Erdfelder E, Lang A-G, Buchner A. G*Power 3: a flexible statistical power analysis program for the social, behavioral, and biomedical sciences. Behav Res Methods. 2007;39:175-91.

22. Choi Y, Choi HS, Jeon WK, et al. Optimal number of endoscopic biopsies in diagnosis of advanced gastric and colorectal cancer. J Korean Med Sci. 2012;27(1):36-9.

23. Won CS, Cho MY, Kim HS, et al. Upgrade of lesions initially diagnosed as low-grade gastric dysplasia upon forceps biopsy following endoscopic resection. Gut Liver. 2011;5:187-93.

24. Imagawa A, Okada H, Kawahara Y, et al. Endoscopic submucosal dissection for early gastric cancer: results and degrees of technical difficulty as well as success. Endoscopy. 2006;38: 987-90.

25. Chung IK, Lee JH, Lee SH, et al. Therapeutic outcomes in 1000 cases of endoscopic submucosal dissection for early gastric neoplasms: Korean ESD Study Group multicenter study. Gastrointest Endosc. 2009;69:1228-35. 